\title{
Pulsating stars as stellar population tracers
}

\section{Gisella Clementini}

\author{
INAF - Osservatorio Astronomico, \\ Via Ranzani n. 1, 40127 Bologna, Italy \\ email: gisella.clementini@oabo.inaf .it
}

\begin{abstract}
Pulsating stars of different types are in different evolutionary phases, thus allowing one to trace stellar components of different age in the host systems. The light variation caused by the cyclic expansion/contraction of the surface layers makes a pulsating star much easier to identify than constant stars in the same evolutionary phase. Pulsating stars thus offer a powerful tool to disentangle the various stellar generations in systems where stars of different age and metal abundance populate the same regions of the colour-magnitude diagram. An overview is presented of how pulsating stars can be used as tools to study the stellar populations, and the structure and formation process in Local Group galaxies.
\end{abstract}

Keywords. stars: oscillations, stars: variables: Cepheids, stars: variables: RR Lyrae, stars: variables: other, galaxies: stellar content, galaxies: structure

\section{Introduction}

A number of virtues make the pulsating stars optimal tools in astrophysics. Their pulsation characteristics (period, amplitudes, pulsation modes) can be determined with great precision, are unaffected by distance and reddening, and are directly linked to the stellar mass, effective temperature, and chemical composition (helium abundance and metallicity), thus allowing one to infer these quantities directly from the pulsation. For instance, the reproduction of the observed light and radial velocity curves of classical Cepheids (CCs; Wood et al. 1997, Bono et al. 2002), RR Lyrae stars (Di Fabrizio et al. 2002, Marconi \& Clementini 2005), and $\delta$ Scuti variables (McNamara et al. 2007) with nonlinear convective pulsation models allows one to predict the variation of the relevant quantities along a pulsation cycle and provides a direct estimate of the star's intrinsic parameters and distance. Recently, Marconi et al.'s (2013) application of this technique to the light and radial velocity curves of CEP0227, a CC in a double-lined eclipsing binary in the LMC (Pietrzyński et al. 2010), provided intrinsic stellar parameters in excellent agreement with the dynamical estimates. On the other hand, the superb light curves produced by the CoRoT and Kepler satellites have revealed that an extraordinary large number of frequencies are excited in pulsating stars, thus allowing the determination of the physical parameters through asteroseismology.

The most common types of pulsating variables, the RR Lyrae stars and the Cepheids, are primary stellar distance indicators in the Local Group (LG) and beyond, up to about $20 \mathrm{Mpc}$. The RR Lyrae stars are standard candles because they obey an absolute magnitude-metallicity relation in the visual band and a period-luminosity-metallicity relation in the $K$-band; the Cepheids are used as standard candles through the periodluminosity, period-luminosity-colour, and the period-Wesenheit (Madore 1982) relationships. The role of these standard candles in setting the basis of the whole astronomical distance scale will be revolutionized by Gaia, the ESA's cornerstone mission scheduled for launch at the end of 2013. The Gaia satellite (Lindegren \& Perryman 1996, Lindegren 2010, Brown 2013) is expected to discover and measure parallaxes with $\sigma_{\pi} / \pi<$ 
$10 \%$ for about 9000 CCs in the Milky Way (MW), as well as to observe more than 70000 RR Lyrae stars in the Galactic halo (Turon et al. 2012). By providing a direct measure of distance to local primary distance indicators via parallax, Gaia will allow a re-calibration of the secondary indicators and a re-assessment of the whole distance ladder, with significant improvement in our knowledge of the Hubble constant.

Pulsating stars of different types are in different evolutionary phases, thus identifying stellar components of different age within the host system. The spatial distribution of the different types of pulsating stars allows the main structures of the parent galaxy to be revealed, and their pulsation properties can provide hints on the mechanisms through which large galaxies have formed. In the following, examples are presented of the use of pulsating stars as tools to trace the different stellar populations, to study the geometric structure, and to derive information on the formation process of the parent galaxies.

\section{Pulsating stars as stellar population tracers}

The colour-magnitude diagram (CMD) is the tool commonly used to study the stellar populations and the star formation (SF) history of a galaxy. However, in the case of remote systems for which only the bright portion of the CMD is observable (with presentday facilities, basically systems beyond a few $\mathrm{Mpc}$ ), it is not easy to prove the existence of $a \geqslant 10$ Gyr-old stellar component. Furthermore, even when the CMD is deep enough to reach the main-sequence turnoff (MSTO) of the oldest population, it may be difficult to disentangle an old component if the system is predominantly composed of young stars and crowding is an issue. Pulsating stars offer a powerful alternative tool to trace stars of different age in a galaxy, as variables of different types arise from parent populations of different age. This property, combined with the advantage of being a pulsating star that is much easier to reveal than a constant star in the same evolutionary phase, makes the pulsating variables invaluable tools specifically in the identification and characterization of the oldest stellar populations in galaxies.

CCs are young $(t \lesssim 100 \mathrm{Myr})$, metal-rich, Population I giants $\left(\log L / L_{\odot} \sim 3-5\right.$, spectral type $\mathrm{F} 6-\mathrm{K} 2, M \sim 3-13 M_{\odot}$ ) that cross the classical instability strip (IS) during the blue-loop phase of the evolutionary tracks. They are preferentially located in gas-rich, dusty, star-forming regions, like galactic bars and spiral arms. Conversely, the RR Lyrae stars are low-mass $\left(M<1 M_{\odot}\right)$ horizontal-branch $(\mathrm{HB})$ giants $\left(\log L / L_{\odot} \sim 1.5-1.8\right.$, A2-F2 spectral type), and along with the Population II Cepheids (P2Cs; low-mass: $M \sim 0.5 M_{\odot}$, post-HB giants: $\log L / L_{\odot} \sim 2$, of F2-G6 spectral type), unambiguously trace the oldest $(t \geqslant 10 \mathrm{Gyr})$ stellar generations commonly found in galactic halos. With their mere presence the RR Lyrae stars and the P2Cs provide evidence that a SF episode occurred more than 10 Gyr ago (see, e.g., the case of the NGC 6822, Clementini et al. 2003). Furthermore, being roughly 3 magnitudes brighter than the coeval MSTO stars, they are much easier to observe, thus allowing one to trace the SF history back to the first epochs of galaxy formation even for systems where the MSTO is unreachable with present-day facilities. Finally, the ACs $\left(\log L / L_{\odot} \sim 1.8-2.3\right.$, spectral type F2-G6, and $M \sim 1.3-2.2 M_{\odot}$ ) are giants evolving form the Zero-Age Horizontal Branch turnover (see Marconi et al. 2004, and references therein) for metallicity lower than $[\mathrm{Fe} / \mathrm{H}] \lesssim-1.7$ dex. They are generally believed to trace an intermediate-age component produced by a relatively recent (a few billion years ago) SF episode, but in principle they could also derive from merging of low-mass, old population stars.

Observations show that RR Lyrae stars are found in all LG galaxies where they are searched for, irrespective of the galaxy morphological type. This provides evidence for a first epoch of SF that is common to all galaxies in the LG, both star-forming and presently quiescent. Observations also show that more massive LG systems (spirals, irregulars) 


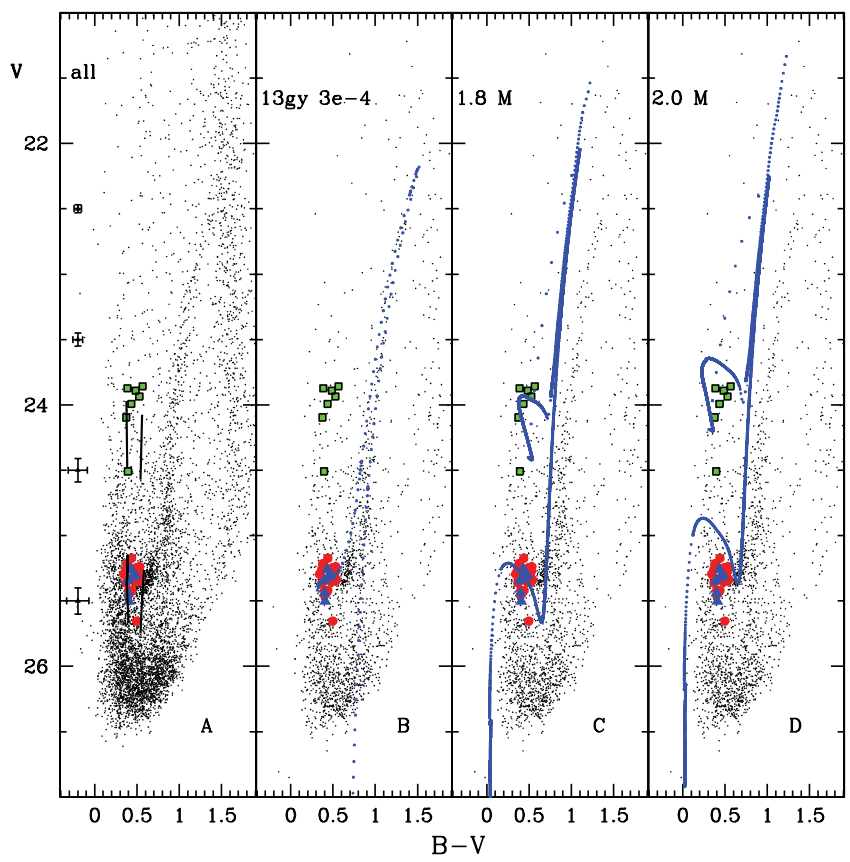

Figure 1. Panel A: Colour-magnitude diagram (CMD) of And XIX from Cusano et al. (2013). Filled circles and triangles are fundamental-mode (RRab) and first-overtone (RRc) RR Lyrae stars, respectively, filled squares are Anomalous Cepheids (ACs). Black solid lines show the boundaries of the instability strip for RR Lyrae stars and ACs with $Z=0.0002$, from Marconi et al. (2004). Panel B: Observed CMD with overlaid stellar isochrones from Padova evolutionary models for $t=13 \mathrm{Gyr}$ and $Z=0.0003$, that well reproduce the galaxy's oldest stellar component traced by the RR Lyrae stars. Panels $C$ and $D$ : Stellar evolutionary tracks from the Basti web site for masses of $1.8 M_{\odot}($ panel $C)$ and $2.0 M_{\odot}($ panel $D)$ that well reproduce the galaxy ACs.

generally contain both CCs (Population I) and RR Lyrae stars (Population II). This suggests that a continuing SF has occurred in these systems. Lower-mass galaxies (mainly dwarf spheroidal galaxies - dSphs) host RR Lyrae stars and ACs (intermediate-age population), thus pointing to mainly early ( $10 \mathrm{Gyr})$ and intermediate-age (a few Gyrs) SF events (see, e.g., the case of Carina, Dall'Ora et al. 2003). Conversely, metal-poor low-mass irregulars host mainly $\mathrm{CCs}$ and ACs, hence pointing to prevalently recent SF episodes. These general trends are followed also by the ultra-faint dwarf (UFD) galaxies recently discovered around the MW (see, e.g., Belokurov et al. 2006, 2010, and references therein) and by the Andromeda's dSph satellites. Tables 1 and 2 summarize the number and type of pulsating stars identified in the MW UFDs and in the M31 dSphs that have been studied for variability.

Figure 1 shows results from the study of the variable stars in Andromeda XIX (And XIX) by Cusano et al. (2013) who have identified 31 RR Lyrae stars (23 RRab and 8 RRc pulsators) and 8 ACs in this new M31 satellite. Panel A shows the galaxy CMD with filled circles and triangles marking RRab and RRc stars, respectively, and filled squares showing the ACs. In Panel B on the CMD are overlaid stellar isochrones from the Padova evolutionary models for $t=13 \mathrm{Gyr}$ and $Z=0.0003$ (from the CMD 2.5 web interface, available at http://stev.oapd.inaf.it/cgi-bin/cmd), that very well reproduce the galaxy's oldest stellar component traced by the RR Lyrae stars. In Panels C and D on the CMD are overlaid stellar evolutionary tracks for masses of $1.8 M_{\odot}\left(\right.$ panel C) and $2.0 M_{\odot}$ (panel 

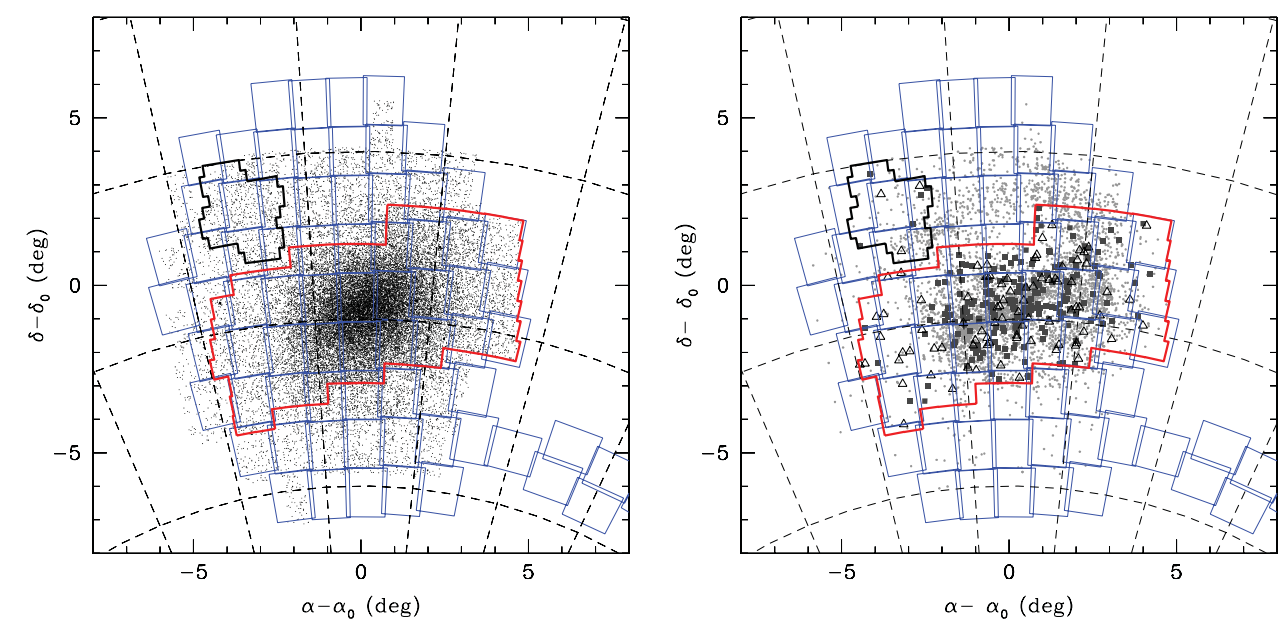

Figure 2. Distribution of RR Lyrae stars (left panel, black points) and Cepheids (classical: grey points, anomalous: open triangles, Population II: filled squares, in the right panel) in the LMC, according to the OGLE III, OGLE IV and EROS-2 surveys. $\alpha_{0}=81.0^{\circ}$ and $\delta_{0}=-69.0^{\circ}$.

D), from the Basti web site (http://albione.oa-teramo.inaf.it/), that well reproduce the And XIX's ACs.

\section{The Large Magellanic Cloud geometry as traced by classical Cepheids and RR Lyrae stars}

Different types of pulsating stars are differently located in space within the parent galaxy; thus they probe its three-dimensional structure and also allow the identification of substructures which are the signature of past interactions between the parent galaxy and its satellites. For instance, the local overdensity of RR Lyrae stars detected in the Galactic halo by Vivas et al. (2001) turned out to be the northern tidal stream of the Sagittarius dSph dissolving itself into the MW halo. Several substructures in the Galactic halo (e.g., the Pisces Overdensity at about $80 \mathrm{kpc}$, Watkins et al. 2009) have been revealed by overdensities of RR Lyrae stars in the Sloan Digital Sky Survey stripe 82 region (see also Sesar et al. 2010). Similarly, evidence for a MW tidal stream reaching beyond $100 \mathrm{kpc}$ and overlapping the Sagittarius stream has been found from the analysis of more than a thousand RR Lyrae stars observed by the Catalina Surveys Mount Lemmon telescope (Drake et al. 2013).

An excellent example of how well different types of pulsating stars can trace the geometrical structure of a galaxy is represented by the Large Magellanic Cloud (LMC). The search for microlensing events started at the beginning of the nineties by the MACHO, EROS and OGLE collaborations has led to the discovery of tens of thousands RR Lyrae stars and Cepheids in the LMC (see e.g., Alcock et al. 1996, Sasselov et al. 1997, Udalski et al. 1997). At present, the largest spatial coverage of the LMC is provided by the second generation of the EROS microlensing experiment (EROS-2; Tisserand et al. 2007). In Fig. 2 we compare the structure of the LMC as traced by the system RR Lyrae stars (left panel) and Cepheids (right panel), combining results for the LMC variables from the OGLE and the EROS-2 surveys. In each panel of the figure thin (blue in the on-line version) rectangles show the tiles of the VISTA Magellanic Clouds Survey (VMC; Cioni et al. 2011), a near-infrared $\left(Y, J, K_{\mathrm{s}}\right)$ ESO public survey of the Magellanic System (MS) which is obtaining multi-epoch photometry in the $K_{\mathrm{s}}$-band to study the MS threedimensional geometry with the pulsating stars. The thick grey (in red on-line) and black 
lines delimit instead the regions where identification of the variables comes from the OGLE III (Soszyński et al. 2008, 2009) and OGLE IV (Soszyński et al. 2012) surveys, respectively. Outside these fields, data for the variables are provided only by the EROS-2 survey. The distributions of RR Lyrae stars (black points in the left panel of Fig. 2) and CCs (grey points in the right panel of Fig. 2) appear to differ significantly. The RR Lyrae stars have a larger density in the central region of the LMC; however, they are present in large numbers also in the peripheral areas covered mainly by EROS-2. Their distribution is smooth and likely traces the LMC halo. Conversely, the CCs are strongly concentrated towards the LMC bar and seem to almost disappear moving outside the region covered by the OGLE III observations (thick grey contours in Fig. 2). However, the larger field covered by EROS-2 reveals the existence of an overdensity of CCs displaced about 2 degrees above the central bar, and running almost parallel to it, to which it connects at its western edge (see, Moretti et al. 2013 for details). This overdensity is likely the signature of a northwest spiral arm of the LMC (see also, Schmidt-Kaler 1977, Nikolaev et al. 2004). The distribution of CCs in the OGLE IV GSEP field (thick black contours in Fig. 2) also shows an increase in the southeastern part of the field, confirming the overdensity of Cepheids highlighted by the EROS-2 data. This demonstrates how pulsating stars can be used to disentangle the fine structure of the LMC.

\section{The Oosterhoff type of the new Milky Way and M31 satellites}

Hierarchical merging and accretion of satellites is the mechanism through which large galaxies are predicted to form in the framework of the $\Lambda$-cold-dark-matter theory. Leftovers of this cannibalistic process are the streams and disrupted/tidally distorted satellites that we observe today within the halo of the MW and in M31. The pulsation properties of the oldest variables, the RR Lyrae stars, may allow one to identify the "building blocks" that have contributed early on to this merging process. In fact, if the MW and M31 have formed by accretion of systems resembling their present-day dwarf satellites, the RR Lyrae stars in these galaxies should have the same characteristics as the variables observed in the MW and M31 halos. A specific property of the RR Lyrae stars in the Galactic globular clusters (GGCs) is the division into two distinct groups based on the mean period of the RRab pulsators $\left\langle P_{\mathrm{ab}}\right\rangle$ (Oosterhoff 1939), and the specific number of fundamental to first-overtone pulsators $f_{c}$. Oosterhoff I (Oo I) GCs have $\left\langle P_{\mathrm{ab}}\right\rangle=$ $0.55 \mathrm{~d}$ and $f_{c} \sim 0.17$. Oo II clusters have $\left\langle P_{\mathrm{ab}}\right\rangle=0.64 \mathrm{~d}$ and $f_{c} \sim 0.44$. The GGC distribution is bimodal with probability larger than 99.99\% (Catelan 2009, and references therein). An Oosterhoff dichotomy is also observed among the MW field RR Lyrae variables (Kinemuchi et al. 2006, Miceli et al. 2008, Drake et al. 2013), where the RR Lyrae stars belong predominantly to the Oo I group, but with a significant Oo II component. The Oosterhoff types of the bright dSph satellites of the MW are summarized in Table 3 of Clementini (2010), while Table 1 summarizes the Oosterhoff type of the MW UFDs. The more massive dSphs around the MW generally have Oo-Intermediate (Oo-Int) properties, thus they do not resemble the "building blocks" of the MW halo. Conversely, the UFDs generally have Oo II type, hence they may resemble the protogalactic fragments from which the MW formed early on.

We still lack a census of the M31 RR Lyrae stars. Brown et al. (2004) identified 55 of these variables in an ACS@HST halo field at $11 \mathrm{kpc}$ from the M31 center and found them to have an Oo-Int type. Sarajedini et al. (2009) detected about 700 RR Lyrae stars in two ACS fields near M32 about 4-6 kpc from Andromeda's center, and conclude that the M31 spheroid has Oo I properties. Finally, Jeffery et al. (2011) discovered 108 RR Lyrae stars in six ACS fields located in the disk, stream and halo of M31 and conclude that the M31 RR Lyrae have Oo I and Oo-Int properties. While none of these studies detected an Oo II 
Table 1. Number of pulsating variables and Oosterhoff properties of Milky Way UFDs

\begin{tabular}{|c|c|c|c|c|c|c|c|}
\hline Galaxy & $\mid \begin{array}{c}\langle[\mathbf{F e} / \mathbf{H}]\rangle^{(1)} \\
\operatorname{dex}\end{array}$ & $\begin{array}{l}\mathbf{N}(\mathbf{R R}) \\
(\mathrm{ab} / \mathrm{c} / \mathrm{d})\end{array}$ & $\mathrm{N}(\mathrm{AC})$ & $\left|f_{c}^{(2)}\right|$ & $\begin{array}{l}\left\langle P_{\mathrm{ab}}\right\rangle \\
\text { day }\end{array}$ & Oо Type $\mid$ & Reference \\
\hline Bootes I & -2.55 & $7 / 7 / 1$ & & 0.53 & 0.69 & Oo II & $\begin{array}{c}\text { Siegel (2006) } \\
\text { Dall'Ora et al. }(2006)\end{array}$ \\
\hline Canes Venatici I & -1.98 & $18 / 5$ & 3 & $|0.22|$ & 0.60 & Oo Int | & Kuehn et al. (2008) \\
\hline $\mid$ Canes Venatici II | & -2.21 & $1 / 1$ & 1 & 1 & 0.74 & Oo II & Greco et al. (2008) \\
\hline Coma & -2.60 & $1 / 1$ & 1 & 1 & 0.67 & Oo II & Musella et al. (2009) \\
\hline Leo IV ${ }^{(5)}$ & -2.54 & 3 & | & 1 & 0.66 & Oo II & Moretti et al. (2009) \\
\hline Ursa Major II & -2.47 & 1 & 1 & 1 & 0.66 & Oo II & Dall'Ora et al. (2012) \\
\hline Ursa Major I & -2.18 & $5 / 2$ & 1 & $|0.29|$ & $0.63 / 0.60$ & Oo Int & Garofalo et al. (2013) \\
\hline Hercules & -2.41 & $6 / 3$ & 1 & $|0.33|$ & 0.68 & Oo II & Musella et al. (2012) \\
\hline | Leo T & -1.99 & 1 & 11 & 1 & 0.60 & Oo Int & | Clementini et al. (2012) \\
\hline
\end{tabular}

Notes:

(1) Metallicities are from the compilation of McConnachie (2012).

${ }^{2} f_{c}$ is the ratio of number of RRc $(\mathrm{Nc})$ to total number of RR Lyrae stars $[\mathrm{N}(\mathrm{ab}+\mathrm{c})]: f_{c}=\mathrm{Nc} / \mathrm{N}(\mathrm{ab}+\mathrm{c})$. In the table we list the $f_{c}$ values only of UFDs containing more than 5 RR Lyrae stars.

(3) Bootes I hosts also a candidate long-period variable.

${ }^{(4)}$ Coma contains also a short-period variable with $P=0.125 \mathrm{~d}$.

(5) Leo IV contains also an SX Phoenicis star.

Table 2. Number of pulsating variables and Oosterhoff properties of M31 dSph satellites

\begin{tabular}{|c|c|c|c|c|c|c|c|}
\hline Galaxy & $\mid \begin{array}{c}\langle[\mathbf{F e} / \mathbf{H}]\rangle^{(1)} \\
\operatorname{dex}\end{array}$ & $\begin{array}{l}\mathbf{N}(\mathbf{R R}) \\
(\mathrm{ab} / \mathrm{c} / \mathrm{d})\end{array}$ & $\mathrm{N}(\mathrm{AC})$ & $f_{c}$ & \begin{tabular}{c|}
$\left\langle P_{\mathrm{ab}}\right\rangle$ \\
day
\end{tabular} \mid & Oо Type & Reference \\
\hline And I & -1.45 & $72 / 26$ & $1^{(2)}$ & $|0.27|$ & 0.575 & Oo I/Oo Int & Pritzl et al. (2005) \\
\hline And II & -1.64 & $64 / 8$ & 1 & $|0.11|$ & $|0.571|$ & Oо I & Pritzl et al. (2004) \\
\hline And $\mathrm{III}^{(3)}$ & -1.78 & $39 / 12$ & 4 & 0.24 & 0.657 & Oo II & Pritzl et al. (2005) \\
\hline And $V$ & -1.6 & $7 / 3$ & & 0.30 & $0.685 ?$ & Oo II? & $\begin{array}{l}\text { Mancone \& Sarajedini (2008) } \\
\text { Sarajedini }(2010) \text {, private com. }\end{array}$ \\
\hline And VI & -1.3 & $91 / 20$ & 6 & $|0.18|$ & 0.588 & Oo Int & Pritzl et al. (2002) \\
\hline And IX & -2.2 & $>30$ & | & | & & 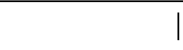 & Ripepi et al., in prep. \\
\hline And $X$ & -1.93 & $9 / 6$ & | & $|0.40|$ & 0.71 & Oo II & Coppola et al., in prep. \\
\hline And XI & -2.0 & $10 / 5$ & | & $|0.33|$ & 0.62 & Oo Int & Yang \& Sarajedini (2011) \\
\hline And XIII & -1.9 & $12 / 5$ & & 0.30 & 0.66 & Oo II & $\begin{array}{c}\text { Contreras Ramos et al., in prep. } \\
\text { Yang \& Sarajedini (2011) }\end{array}$ \\
\hline And XVI & -2.1 & $3 / 6$ & | & $|0.67|$ & 0.64 & Oo II & Mercurio et al., in prep. \\
\hline And XIX & -1.9 & $23 / 8$ & 8 & $|0.26|$ & 0.62 & Oo Int & Cusano et al. (2013) \\
\hline And XXI & -1.8 & $37 / 5$ & 8 & $|0.12|$ & 0.63 & Oo II/Oo Int & Garofalo et al., in prep. \\
\hline
\end{tabular}

Notes:

(1) Metallicities are from the compilation of McConnachie (2012).

${ }^{(2)}$ Uncertain classification, this variable could as well be a P2C.

${ }^{(3)}$ And III contains also a P2C.

component in the M31 halo, first studies of the Oosterhoff dichotomy in the M31 GCs (Clementini et al. 2009, Contreras Ramos et al. 2013), showed that both Oo-Int and Oo II types seem to occur. Table 2 summarizes the Oosterhoff type of the M31 dSphs for which this information is currently available, and Fig. 3 shows the period-amplitude diagram 


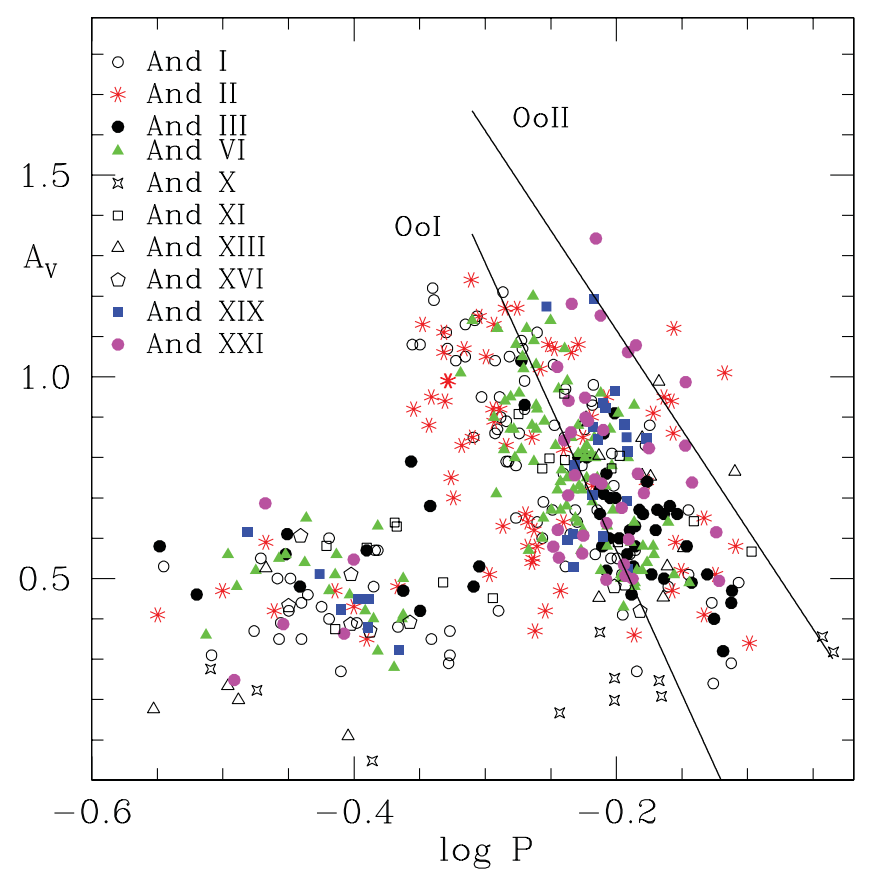

Figure 3. Period-amplitude diagram in the $V$-band for the RR Lyrae stars in the M31 dwarf satellites And I, And II, And III, And VI, And X, And XI, And XIII, And XVI, And XIX and And XXI. Solid lines show the loci defined by Oo I and Oo II GGCs according to Clement \& Rowe (2000).

of the RR Lyrae stars in these systems. According to the $\left\langle P_{\mathrm{ab}}\right\rangle$ values the M31 dSphs analyzed so far are of Oo II and Oo-Int types; however, in the $\log P-A_{V}$ diagram (Fig. 3) these systems appear to be preferentially of Oo I type. Clearly, further investigations are needed to shed light on the Oosterhoff properties of the M31 RR Lyrae stars.

\section{Acknowledgements}

Results presented in this review are largely based on work by undergraduates, PhDs, post-Docs, and young researchers. For this, special thanks go to (in alphabetic order): M. Cignoni, R. Contreras Ramos, G. Coppola, F. Cusano, A. Garofalo, A. Mercurio, M.I. Moretti, and T. Muraveva. It is a pleasure to thank Marcella Marconi and Vincenzo Ripepi for comments on an earlier version of this review.

\section{References}

Alcock, C., Allsman, R. A., Axelrod, T. S., et al. 1996, AJ, 111, 1146

Belokurov, V., Walker, M. G., Evans, N. W., et al. 2010, ApJ, 712, L103

Belokurov, V., Zucker, D. B., Evans, N. W., et al. 2006, ApJ, 647, L111

Bono, G., Castellani, V., \& Marconi, M. 2002, ApJ, 565, L83

Brown, A. 2013, in: L. Cambresy, F. Martins, E. Nuss, \& A. Palacios (eds.), Proc. Soc. Francaise d'Astronomie et d'Astrophysique (SF2 A) 2013, in press, (arXiv: 1310.3485)

Brown, T. M., Ferguson, H. C., Smith, E., et al. 2004, AJ, 127, 2738

Catelan, M. 2009, Ap\&SSS, 320, 261

Cioni M.-R. L., Clementini G., Girardi L., et al. 2011, A\&A, 527, 116

Clement, C. M. \& Rowe, J. 2000, AJ, 120, 2579

Clementini, G. 2010, in: C. Sterken, N. Samus, \& L. Szabados (eds.), Variable Stars, the Galactic Halo and Galaxy Formation, (Moscow: Sternberg Astronomical Institute of Moscow University Publications), p. 107, (arXiv: 1002.1575) 
Clementini, G., Held, E. V., Baldacci, L., \& Rizzi, L. 2003, ApJ, 588, L85

Clementini, G., Contreras, R., Federici, L., et al. 2009, ApJ, 704, L103

Clementini, G., Cignoni, M., Contreras Ramos, R., et al. 2012, ApJ, 756, 108

Contreras Ramos, R., Clementini, G., Federici, L., et al. 2013, ApJ, 765, 71

Cusano, F., Clementini, G., Garofalo, A., et al. 2013, ApJ, 779, 7

Dall'Ora, M., Ripepi, V., Caputo, F., et al. 2003, ApJ, 126, 197

Dall'Ora, M., Clementini, G., Kinemuchi, K., et al. 2006, ApJ, 653, L109

Dall'Ora, M., Kinemuchi, K., Ripepi, V., et al. 2012, ApJ, 752, 42

Di Fabrizio, L., Clementini, G., Marconi, M., et al. 2002, MNRAS, 336, 841

Drake, A. J., Catelan, M., Djorgovski, S. G., et al. 2013, ApJ, 765, 154

Garofalo, A., Cusano, F., Clementini, G., et al. 2013, ApJ, 767, 62

Greco, C., Dall'Ora, M., Clementini, G., et al. 2008, ApJ, 675, L73

Jeffery, E. J., Smith, E., Brown, T. M., et al. 2011, AJ, 141, 171

Kinemuchi, K., Smith, H. A., Woźniak, P. R., \& McKay, T. A. 2006, AJ, 132, 1202

Kuehn, C., Kinemuchi, K., Ripepi, V., et al. 2008, ApJ, 674, L81

Lindegren, L. 2010, in: S. A. Klioner, P. K. Seidelmann, \& M. H. Soffel (eds.), Relativity in Fundamental Astronomy: Dynamics, Reference Frames, and Data Analysis, Proc. IAU Symposium No. 261 (Cambridge: Cambridge University Press), p. 296

Lindegren, L. \& Perryman, M. A. C. 1996, A\&AS, 116, 579

Madore, B. F. 1982, ApJ, 253, 575

Mancone, C. \& Sarajedini, A. 2008, AJ, 136, 1913

Marconi, M. \& Clementini, G. 2005, AJ, 129, 2257

Marconi, M., Fiorentino, G., \& Caputo, F. 2004, $A \& A$, 417, 1101

Marconi, M., Molinaro, R., Bono, G., et al. 2013, ApJ, 768, L6

McConnachie, A. W. 2012, AJ, 144, 4

McNamara, D. H., Clementini, G., \& Marconi, M. 2007, AJ, 133, 2752

Miceli, A., Rest, A., Stubbs, C. W., et al. 2008, ApJ, 678, 865

Moretti, M. I., Clementini, G., Muraveva, T., et al. 2013, MNRAS, in press (arXiv:1310.6849)

Moretti, M. I., Dall'Ora, M., Ripepi, V., et al. 2009, ApJ, 699, L125

Musella, I., Ripepi, V., Clementini, G., et al. 2009, ApJ, 695, L83

Musella, I., Ripepi, V., Marconi, M., et al. 2012, ApJ, 765, 121

Nikolaev, S., Drake, A. J., Keller, S. C., et al. 2004, ApJ, 601, 260

Oosterhoff, P. T. 1939, Observatory, 62, 104

Pietrzyński, G., Thompson, I. B., Gieren, W., et al. 2010, Nature, 468, 542

Pritzl, B. J., Armandroff, T. E., Jacoby, G. H., \& Da Costa, G. S. 2002, AJ, 124, 1464

Pritzl, B. J., Armandroff, T. E., Jacoby, G. H., \& Da Costa, G. S. 2004, AJ, 127, 318

Pritzl, B. J., Armandroff, T. E., Jacoby, G. H., \& Da Costa, G. S. 2005, AJ, 129, 2232

Sarajedini, A., Mancone, C. L., Lauer, T. R., et al. 2009, AJ, 138, 184

Sasselov, D. D., Beaulieu, J. P., Renault, C., et al. 1997, A\&A, 324, 471

Schmidt-Kaler, T. 1977, A\& A, 54, 771

Sesar, B., Ivezić, Ž., Grammer, S. H., et al. 2010, ApJ, 708, 717

Siegel, M. H. 2006, ApJ, 649, L83

Soszyński I., Poleski R., Udalski A., et al. 2008, AcA, 58, 163

Soszyński, I., Udalski, A., Szymanski, M. K., et al. 2009, AcA, 59, 1

Soszyński, I., Udalski, A., Poleski, R., et al. 2012 AcA, 62, 219

Tisserand, P., Le Guillou, L., Afonso, C., et al. 2007, A\& A, 469, 387

Turon, C., Luri, X., \& Masana, E. 2012, Ap\&SSS, 341, 15

Udalski, A., Kubiak, M., \& Szymański, M. 1997, AcA, 47, 319

Vivas, A. K., Zinn, R., Andrews, P., et al. 2001, ApJ, 554, L133

Watkins, L. L., Evans, N. W., Belokurov, V., et al. 2009, MNRAS, 398, 1757

Wood, P. R., Arnold, A., \& Sebo, K. M. 1997, ApJ, 485, L25

Yang, S.-C. \& Sarajedini, A. 2012, MNRAS, 419, 1362 